\title{
Spinal manipulation effect on pain and cardiac autonomic modulation in patients with rotator cuff tendinopathy: a pilot study
}

\author{
Alyssa Conte Silva ${ }^{(1)}$, Juliana Falcão Padilha(2), Jefferson Luiz Brum Marques ${ }^{(3)}$, Cláudia Mirian de Godoy Marques ${ }^{(1)}$
}

\begin{abstract}
Introduction: there are few studies that propose to identify the relationship of Vertebral Manipulation (VM) on pain and cardiac autonomic modulation, especially in subjects with rotator cuff injury. Objective: To analyze the effect of chest VM on the pain and cardiac autonomic modulation in patients with rotator cuff (RC). Method: quasi-experimental study with a quantitative approach. Sample of 6 subjects divided into two groups: Asymptomatic Group $(A G, n=3)$ not presenting shoulder injury and pain complaints and Symptomatic Group (SG, $n=3$ ), with rotator cuff injury. Pain assessment was performed by Visual Analog Scale (VAS) before and after handling, as well as the assessment of Heart Rate Variability (HRV) using an electrocardiogram (ECG) with 8 minutes of duration. Chest VM was held on 4th and 5th thoracic vertebra. The analysis of HRV was performed in the Frequency Domain (VLF-very low frequency, LF-low frequency, HF-high frequency, LF/HF-ratio between low and high frequency). The statistical analysis used the Wilcoxon test for comparison in the same group and the Mann Whitney test for comparison between groups, adopting a $5 \%$ significance ( $p<0.05$ ). Results: There was a reduction of post handling pain, but no statistically significant difference in SG ( $p=0.317)$. In HRV the AG showed a decrease VLF $(p=0.83)$ and increased LF $(p=0.51), \operatorname{HF}(p=0.83)$ and LF/HF $(p=0.51)$ variables. In the SG, there was an increase in VLF $(p=0.83), L F(p=0.83), H F(p=0.51)$ variables and a decrease in LF/HF ( $p=0.51)$ variable. Conclusion: The upper thoracic VM had no effect on the pain and cardiac autonomic modulation in patients with rotator cuff (RC).
\end{abstract}

Keywords: Rotator Cuff; Pain; Shoulder; Spinal Manipulation; Tendinopathy;

\section{INTRODUCTION}

The most frequent cause of shoulder pain is rotator cuff injury, which can affect individuals in any age group, being potentiated with aging and labor or recreational occupation ${ }^{(1)}$. The tendinopathy by this injury may include partial and/or complete tendinopathy of the supraspinatus, infra-spinous and/or subscapularis tendon and may be associated with tendinopathy in the tendon of the long head of the biceps ${ }^{(2)}$.

The patient with rotator cuff tendinopathy (RCT) usually presents reduced shoulder function and may be accompanied by night and resting pain and painful range of motion $^{(3,4)}$. Conservative treatment is initially recommended for the treatment of RCT. In this context, some studies have proposed as treatment for shoulder pain the thoracic vertebral manipulation obtaining clinically positive results for pain and functionality of this joint ${ }^{(5-7)}$.

The mechanism of action of the vertebral manipulation (VM) is not well defined in the literature. However, some authors argue that the VM may stimulate the sympathetic and parasympathetic centers in order to influence the Autonomic Nervous System (ANS) and, consequently, the autonomic modulation ${ }^{(8,9)}$, which may be altered in people who have some dysfunction and / or pain ${ }^{(10,11)}$.

One of the measures to verify the autonomic modulation is through the Heart Rate Variability (HRV) measured by the electrocardiogram (ECG). This corresponds to the change in the interval or distance between one heart beat and the next one, providing the balance between the sympathetic and parasympathetic system ${ }^{(12)}$.

Based on the above, this study aims to investigate the effect of thoracic VM on pain and autonomic cardiac modulation in individuals with RCT.

\section{METHOD}

It is a quasi-experimental pilot study with a quantitative approach. The research was approved by the Ethics and Research Committee on Human Beings of the "Universidade do Estado

Corresponding author: Cláudia Mirian de Godoy Marques (Universidade do Estado de Santa Catarina - UDESC/ Centro de Ciências da Saúde e do Esporte - CEFID/ Departamento de Ciências da Saúde). Rua Paschoal Simone, 358 - Coqueiros; Florianópolis - SC; Brazil, CEP 88080-350. E-mail: claudia.marques@udesc.br

(1) Universidade do Estado de Santa Catarina (UDESC), Florianópolis (SC), Brazil.

Full list of author information is available at the end of the article.

Financial support: The authors declare that there was no financial support.

Submission date 21 September 2016; Acceptance date 4 December 2016; Publication online date 22 December 2016 
de Santa Catarina" (UDESC) under the Ethical Appreciation Certificate (CAAE) number 37088014.0.0000.0118. All subjects signed the Free and Informed Consent Form to participate in the study.

The subjects were divided into two groups: Asymptomatic Group ( $A G$ ), without lesion and painful complaint in the shoulder and Symptomatic Group (OS) presenting pain and rotator cuff lesion.

The process of selection of the participants was intentional, with SG inclusion criteria: individuals with rotator cuff tendinopathy of both genders, aged between 20 and 70 years, presenting pain for at least 6 months, who accepted to be submitted to kinetic-functional evaluation, to present a medical diagnosis and/or image of rotator cuff lesion, not to be in physiotherapeutic treatment and not to be using anti-inflammatory drugs and beta-blockers for at least 1 month. For GA, the same criteria were adopted, but with no lesion in the shoulder and pain.

The exclusion criteria considered participants with complete rotator cuff lesion who underwent surgical intervention on the shoulders, presented absolute contraindication for vertebral manipulation (fractures, severe osteoporosis, malignancy, circulatory disorders such as aneurysms, anti-coagulant therapy, atherosclerosis, rheumatoid arthritis (acute phase), spondylolisthesis, vertebral dislocation), painful spinal complaints (chest region), history of surgery or trauma in the spine, pregnant women, history of cancer, neurological disease and visual and/or auditory.

Initially, an anamnesis record was made up using the identification data of each participant and questions pertinent to the research, such as age, gender, shoulder that presented the lesion among others. Subsequently, the kinetic-functional evaluation was performed by a physiotherapist with 5 years of experience in the area to prove the lesion in question. This assessment consisted of five clinical trials, in which the individuals should present at least three with positive results, thus indicating signs of rotator cuff injury ${ }^{(13)}$. The tests were: (1) Positive Hawkins test; (2) Positive Neer test; (3) Pain during active elevation less than 60 degrees on the scapula plane or the sagittal planel; (4) Positive Jobe Test; (5) Pain or weakness with resisted external rotation of the shoulder with the arm at the side of the body.

Subsequently, the participants were assessed for pain through the Visual Analogue Scale (VAS) before and after manipulation. VAS consists of a scale for assessing the level of pain. In this scale, there is a horizontal line of $10 \mathrm{~cm}$ with the number 0 (zero) marked at the left of the line and number 10 (ten) marked at the right of the same line, and 0 corresponding to no pain and 10 to maximum pain ${ }^{(14)}$. Thus, the individuals made a mark in the line to demarcate the level of pain and the evaluator measured with a ruler from the mark 0 to the trait marked by the subjects. This evaluation was recorded in the anamnesis form of each participant.
Afterwards, the evaluation of the autonomic modulation by HRV was performed using an electrocardiogram (ECG) with duration of 8 minutes before and after manipulation. The subjects were placed in dorsal decubitus on a stretcher, asking them to remain in rest, immobile, without speaking and to maintain the habitual breathing. Three disposable ECG electrodes were placed in the thorax of participants in lead II. In this way, the first electrode was located on the right subclavian space (negative terminal), the 2 nd located on the 8th left intercostal space (positive terminal) and 3 o on the 9th right intercostal space (reference terminal).

After the first recording, the volunteers positioned themselves in the ventral decubitus (VD) to receive the VM. The VM was performed on the thoracic spine (4th and 5th thoracic vertebrae), using the technique called "Pisiformes Cruzados". The participants were placed in the ventral position with the upper limbs along the body. The physiotherapist was orthostatic to the right of the participant, at the height of the thoracic spine and made contact with the hypothenar eminence of the right and left hand in the transverse processes, firstly of the 4th thoracic vertebra, keeping the upper limbs in extension. The therapist exerted pressure on the vertebra, keeping its upper limbs in extension and asked the participant to take a deep breath and made an impulse at the end of the expiration. The technique was applied perpendicularly and parallel to the joint plane ${ }^{(16)}$. After manipulation of the 4 th thoracic vertebra, the same procedure was performed for the manipulation of the 5th thoracic vertebra. Afterwards, individuals returned to the initial position and the time of 1 minute for stabilizing the volunteer's posture was respected, occurring than the second recording of ECG (post-).

The signal capture was carried out through the surface electrodes connected to an ECG amplifier using a hardware developed by the "Instituto de Engenharia Biomédica da Universidade Federal de Santa Catarina". The acquisition software named DATAQ Instruments Hardware Manager was used. Thus, data collection was obtained through a cardiac monitor and scanned with a 12-bit analog-to-digital converter at a sampling frequency of $1000 \mathrm{~Hz}$ and the resulting signal was digitally filtered (range 10 to $40 \mathrm{~Hz}$ ).

The ECG signals were digitally processed for HRV analysis and for the HRV quantification were used linear methods in the Frequency Domain (FD). In this one, spectral analysis was used, being the Fast-Fourie Transform method used to calculate the Spectral Density of Power. The HRV variables were analyzed in milliseconds squared $\left(\mathrm{ms}^{2}\right)$ and to all the variables a logarithmic (Log) transformation was applied.

Thus, the variables in the FD verified were: VLF (variability of the RR interval in the very low frequency range - with variation between 0,003 and $0,04 \mathrm{~Hz}$ - reflecting the parasympathetic activity), LF (variability of the RR intervals in the low frequency range - with variation between 0,04 and $0,15 \mathrm{~Hz}$ - reflecting the predominant manifestation of sympathetic nervous 
system), HF (variability of the RR interval in the high frequency range - with variation between 0,15 and $0,4 \mathrm{~Hz}$ - reflecting the predominant manifestation of parasympathetic SNA) and LF / HF (ratio of LF and HF components reflecting the sympatho-vagal balance) ${ }^{(15)}$.

Statistical analysis was performed by the software SPSS 2.0 version. The data normality was tested by Shapiro-Wilk. To compare the means (pre versus post) of pain and intra-group HRV, was used the Wilcoxon test and for the comparison between groups, was used the Mann Whitney test. For all tests was considered a significance level of $5 \%$.

\section{RESULTS}

The study sample was composed of 6 volunteers: $A G n=3$ ( 1 male and 2 females; mean age of 55,3 years) and SG $n=3$ ( 1 male and 2 females; mean age of 38,6 years).

The mean time that SG presented pain was 4,3 years. The mean pain verified by the VAS before the manipulation was 4 and after was 3,3, showing no statistically significant difference ( $p=0,317)$ (Figure 1 ).

Regarding the HRV in AG after thoracic VM, there was a decrease in the VLF variable, and an increase in the LF and HF variables, but there was no statistically significant difference (Figure 2).

In SG after thoracic VM, there was an increase in VLF, LF and $\mathrm{HF}$ variables, but there was no significant difference (Figure 3 ).
The LF/HF ratio between the groups was already different in the pre-manipulation condition. The AG presented lower value in the pre-manipulation situation in relation to the SG. After manipulation, the LF/HF ratio in AG increased, while SG decreased, however without significant difference between groups and in the same group (Figure 4).

\section{DISCUSSION}

The present study, although there was no significant difference, showed reduction of pain after thoracic VM. This result is in line with another study in which shoulder pain was verified in 30 individuals with signs of RCT in pre and post thoracic manipulation, as well as the assessment of scapular kinematics performed with a three-dimensional apparatus, electromyographic analysis of the musculature and shoulder function. As a result there was immediate improvement in pain after vertebral manipulation and no significant differences were found in shoulder kinematics or shoulder muscle activity, suggesting that pain reduction could not be explained by these factors. The authors also cite as an important limitation of this study the inexistence of a placebo group or control group and therefore cannot establish a cause and effect relationship ${ }^{(17)}$.

Likewise, the present study did not include a placebo group. Therefore, despite the sensation of reduction of the painful situation (although without significant difference), the possibility of the placebo effect is not ruled out ${ }^{(18)}$, since this

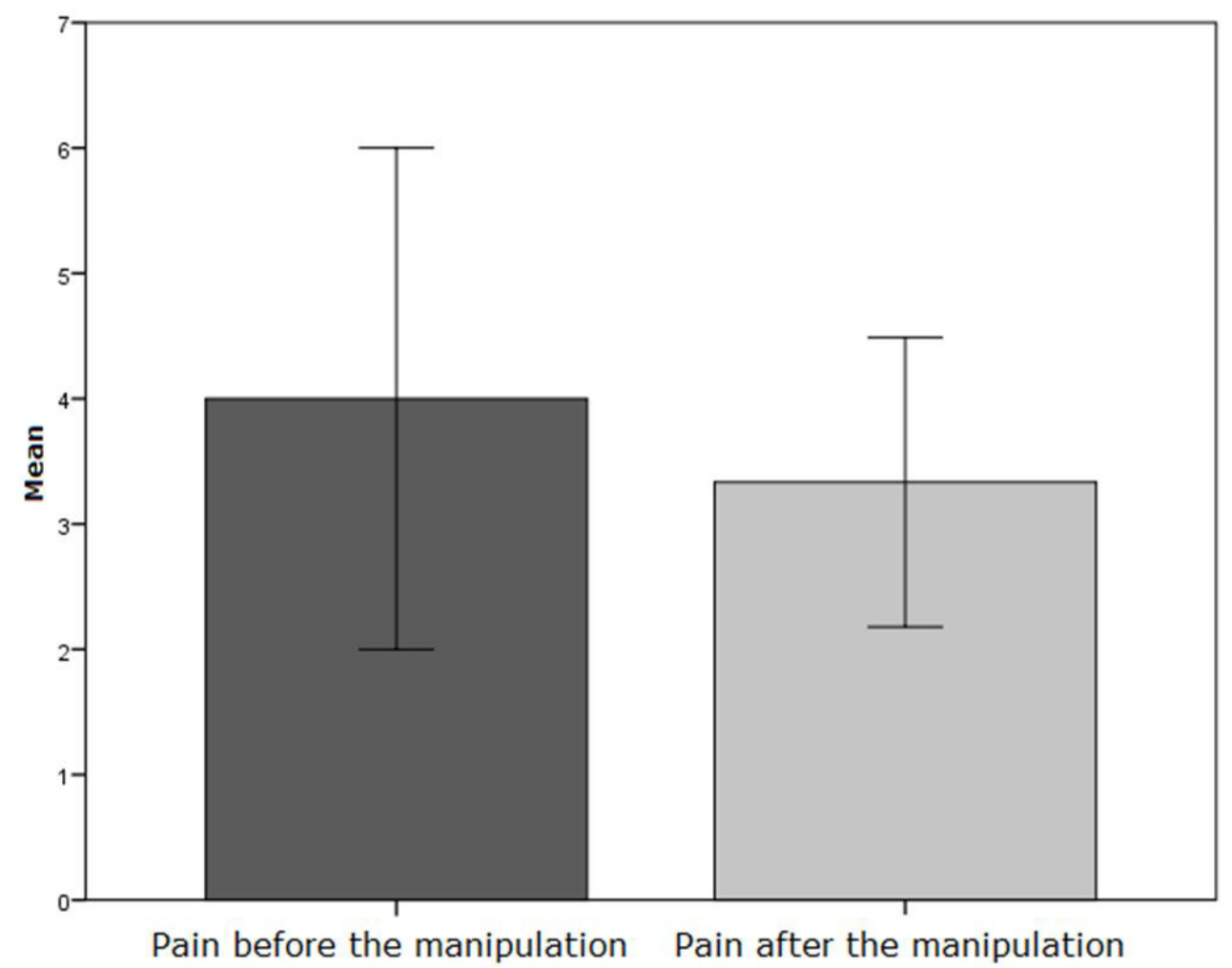

Figure 1. Assessment of pain before and after thoracic vertebral manipulation in the Symptomatic Group (SG) (values expressed as mean and standard deviation). 
is related to the perception that the actual therapy is being administered $^{(18-20)}$ presenting benefits.

Regarding HRV in SG, it was possible to notice an increase in VLF, HF and LF after thoracic VM, even without significant difference. As in the variables VLF and HF there is predominance of the parasympathetic activity and in

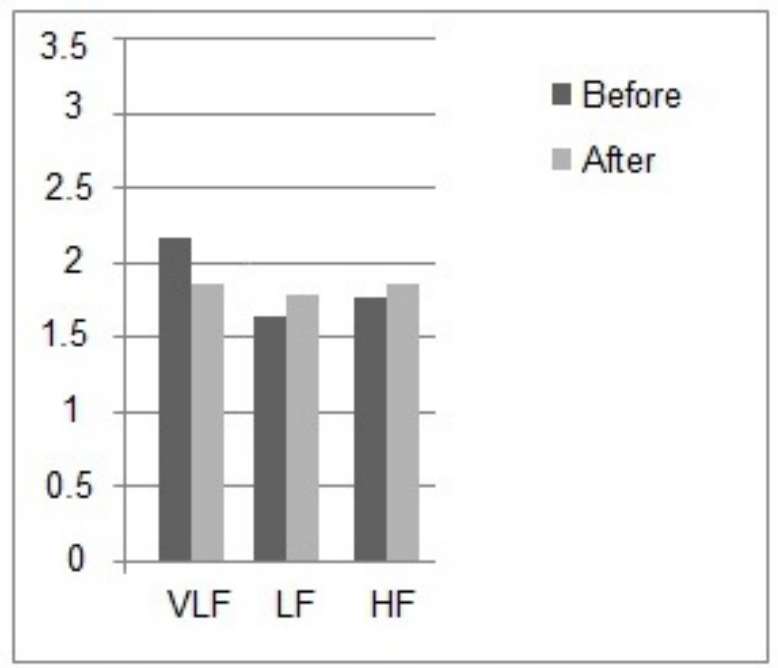

Figure 2. Comparison of the parameters of HRV analysis in the Frequency Domain (VLF, LF and HF) before and after thoracic vertebral manipulation in the Asymptomatic Group (AG) (values expressed in Log of the presented variables). $\operatorname{VLF}(p=0,83) ; \operatorname{LF}(p=0,51) ; \operatorname{HF}(p=0,83) ; \operatorname{VLF}=$ Variability of RR intervals in the very low frequency range; $L F=$ Variability of $R R$ intervals in the low frequency range; $\mathrm{HF}=$ Variability of $\mathrm{RR}$ intervals in the high frequency range.

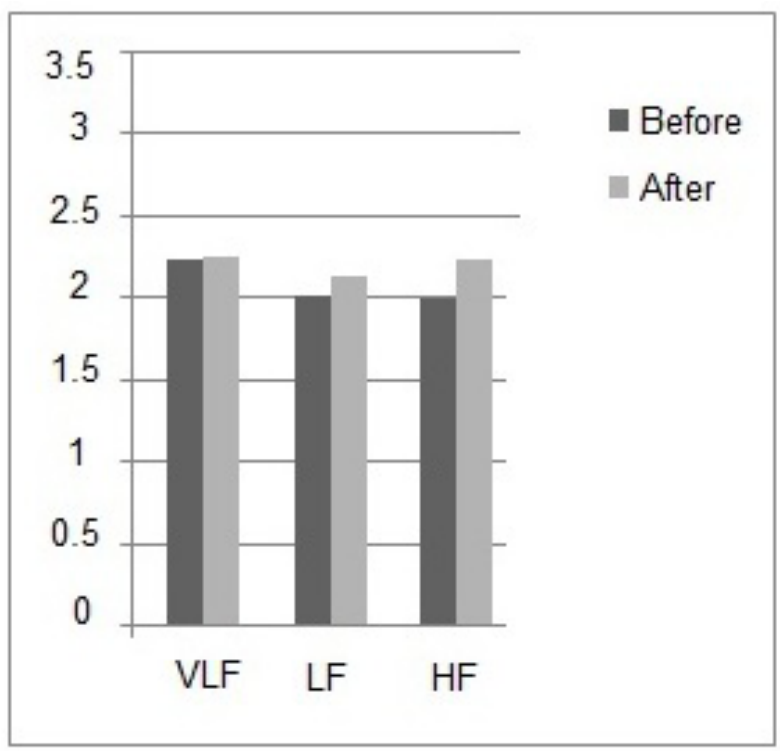

Figure 3. Comparison of the parameters of HRV analysis in the Frequency Domain (VLF, LF and HF) before and after thoracic vertebral manipulation in the Symptomatic Group (SG) (values expressed in Log of the presented variables). $\operatorname{VLF}(p=0,83) ; \operatorname{LF}(p=0,83) ; \operatorname{HF}(p=0,51) . V L F=$ Variability of RR intervals in the very low frequency range; $L F=$ Variability of $R R$ intervals in the low frequency range; $\mathrm{HF}=$ Variability of $\mathrm{RR}$ intervals in the high frequency range
LF sympathetic predominance ${ }^{(15)}$, may be assumed that after manipulation there was greater stimulation of the parasympathetic system in subjects with RCT.

This result goes to the opposite of the literature, since, in theory, a vertebral manipulation in the upper thoracic region would result in stimulation of the sympathetic nervous system. This effect would occur because the cellular bodies of the sympathetic fibers are on the sides of the spinal segments $\mathrm{T} 1$ to $\mathrm{L} 2$. In these regions of the spine, in which sympathetic innervation predominates, manipulation in the upper thoracic region could result in a sympathetic response (e.g. heart rate stimulation, increased blood pressure, and dilated pupils) ${ }^{(21,22)}$, and this feature was not present.

Both groups presented increased LF and HF variables after thoracic VM, although without significant difference. This same autonomic behavior was observed in another study, but with a significant alteration, which it verified the effects of thoracic VM and a simulated procedure on pre and post HRV, showing that manipulation of the thoracic spine was associated to changes in HRV that were not observed by the simulated procedure. This effect was observed by the low frequency (LF) and high frequency (HF) components that increased after the manipulation, whereas the subjects submitted to manipulation of the simulated thoracic spine did not present statistically significant variation $^{(23)}$.

The AG, in comparison to the SG, demonstrated a difference in the autonomic behavior only for the VLF variable, presenting a decrease after the thoracic VM. As previously mentioned, this variable is indicative of parasympathetic activity and can suggest $^{(15)}$, therefore, a decrease in parasympathetic nervous system after manipulation in these individuals, however, with no significance.

Other studies that performed the VM demonstrated a significant change in the VLF variable after manipulation, however, they were subjects with acute low back pain ${ }^{(12)}$, different from the present study in which subjects presented

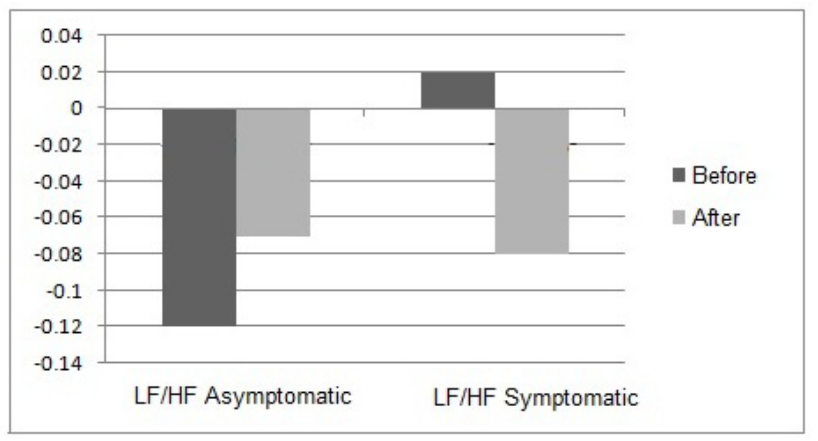

Figure 4. Comparison of the LF / HF ratio between the Asymptomatic Group (AG) and the Symptomatic Group (SG) before and after thoracic vertebral manipulation (values expressed in Log of the presented variables). LF/ HF Assintomático: Asymptomatic LF/HF $(p=0,83)$; LF/HF Sintomático: Symptomatic LF/HF $(p=0,51)$. Pré: Before; Pós: After; LF/HF= Ratio of low frequency and high frequency components. 
chronic pain. In addition, the manipulated region was the lumbar region, which also differs from the present study.

Regarding the LF/HF relationship again no significant difference was observed, but it was possible to perceive between the groups that this was different already in the pre-manipulation situation. The AG presented lower value in the pre-manipulation situation in relation to the SG and after the manipulation, the LF/HF ratio in the AG increased, while in the SG it decreased, however without presenting significant difference between the groups and in the same group.

The balance between these two systems was the target of some studies that used the VM in which was found a difference in LF/HF after manipulation ${ }^{(11)}$. However, in some cases this was identified in case study that made use of a set of vertebral manipulations in the four regions of the spine ${ }^{(24)}$, different from the present study that used only one manipulation technique, destined to a single vertebral level (T4-T5).

Finally, the fact that the present research is a pilot study, we cannot generalize the results found. The data did not show statistically significant differences for comparisons between groups (AG vs SG) or time (before vs after) for pain and for cardiac autonomic modulation most likely due to small sample. However, it was possible to observe a trend in SG to show an increase in parasympathetic activity after manipulation. In this sense, more research is needed to support or refute the findings of the study.

\section{CONCLUSION}

From the present study, the effect of thoracic VM on the pain and autonomic nervous system cannot be observed in the individuals investigated with RCT, since the alterations resulting from VM found were not statistically significant. Therefore, a greater number of participants, the inclusion of a placebo group and blind evaluation of the subjects are suggested for future studies.

\section{AUTHORS' CONTRIBUTIONS}

ACS: conception and design of the study, data collection, analysis and interpretation of data, article writing. JFP: Analysis and interpretation of data, article writing. JLBM: Statistical analysis, interpretation of data. CMGM: Critical review of the manuscript regarding the technical-scientific content, conception and design of the study, statistical analysis.

\section{CONFLICT OF INTEREST}

The authors declare that they have no conflicts of interest.

\section{AUTHOR DETAILS \\ (2) Universidade Federal do Pampa (UNIPAMPA), Uruguaiana (RS), Brazil. \\ (3) Universidade Federal de Santa Catarina (UFSC), Florianópolis (SC), Brazil.}

\section{REFERENCES}

1. Saggini R, Cavezza T, Di Pancrazio L, Pisciella V, Saladino G, Zuccaro $\mathrm{MC}$, et al. Treatment of lesions of the rotator cuff. J Biol Regul Homeost Agents. 2010;24(4):453-9.

2. Lasbleiz S, Quintero N, Ea K, Petrover D, Aout M, Laredo JD, et al. Diagnostic value of clinical tests for degenerative rotator cuff disease in medical practice. Ann Phys Rehabil Med. 2014;57(4):228-43.
3. Fukuda H. Partial-thickness rotator cuff tears: a modern view on Codmans classic. J. Shoulder Elbow Surg. 2000;9(2):163-168.

4. Shin KM. Partial-thickness rotator cuff tears. Korean J Pain. 2011;24(2):6973.

5. Haik M, Sendín F, Silva C, Junior A, Ribeiro I, Camargo P. Scapular Kinematics pré-and post- thoracic thrust Manipulation in individuals with and without shoulder impingement symptoms: a randomized contolled study. J Orthop Sports Phys Ther. 2014;44(7):475-487.

6. Riley S, Bialosky J, Cote M, Swanson B, Tafuto V, Sizer P, et al. Thoracic spinal manipulation for musculoskeletal shoulder pain: Can an instructional set change patient expectation and outcome? Man. Ther. 2015;20(3):469-474.

7. Michener LA, Kardouni JR, Sousa CO, Ely JM. Validation of a sham comparator for thoracic spinal manipulation in patients with shoulder pain. Man Ther. 2015;20(1):171-175.

8. Ricard F, Sallé JL. Tratado de Osteopatía. 3‥ed. Madrid: Panamericana, 2010.

9. Welch A, Boone R. Sympathetic and parasympathetic responses to specific diversified adjustments to chiropractic vertebral subluxations of the cervical and thoracic spine. Journal of Chiropratic Medicine. 2008;7(3):8693.

10. Shafiq $H$, Mcgregor $C$, Murphy B. The impact of cervical manipulation on heart rate variability. Engineering in Medicine and Biology Society. 2014;3406-3409.

11. Roy R, Bouche, J, Comtois A. Heart rate variability modulation after manipulation in pain-free patients vs patients in pain. Journal of Manipulative and Physiological Therapeutics. 2009;32(4):277-286.

12. Moss D, Shaffer F. Heart Rate Variability Training. [Internet]. [Acesso em: 6 de mai. 2016] Disponível em: <http://www.bfe.org/articles/hrv.pdf>.

13. Michener LA, Walsworth MK, Doukas WC, Murphy KP. Reliability and Diagnostic Accuracy of 5 Physical Examination Tests and Combination of Tests for Subacromial Impingement. Arch Phys Med Rehabil. 2009;90(11):1898-903.

14. Tashjian RZ, Deloach J, Porucznik CA, Powell AP. Minimal clinically important differences (MCID) and patient acceptable symptomatic state (PASS) for visual analog scales (VAS) measuring pain in patients treated for rotator cuff disease. J Shoulder Elbow Surg. 2009;18(6):927-932.

15. Task Force of the European Society of Cardiology. Heart rate variability: standards of measurement, physiological interpretation and clinical use. Task Force of the European Society of Cardiology and the North American Society of Pacing and Electrophysiology.Circulation. 1996;93(5):10431065.

16. Maitland G, Hengeveld E, Banks K, English K. Maitland's Vertebral Manipulation. 7으. ed. Philadelphia: Elsevier Butterworth-Heinemann, 2007.

17. Muth S, Barbe M, Lauer R, Mcclure P. The effects of thoracic spine manipulation in subjects with signs of rotator cuff tendinopathy. J Orthop Sports Phys Ther. 2012;42(12):1005-1016.

18. Colloca L, Benedetti F. Placebos and painkillers: is mind as really as matter? Nature Rev Neurosci. 2005;6:545-552.

19. Benedetti F. Mechanisms of placebo and placebo-related effects across diseases and treatments. Annu Rev Pharmacol Toxicol. 2008;48:33-60.

20. Benedetti F. The Patient's Brain: The Neuroscience Behind the DoctorePatient Relationship. Oxford: Oxford University Press, 2010.

21. Bakewell S. The autonomic nervous system. Update Anaesth. 1995;(5):6.

22. Budgell BS. Reflex effects of subluxation: the autonomic nervous system. J Manipulative Physiol Ther. 2000;23(2):104-106.

23. Budgell $B$, Polus $B$. The effects of thoracic manipulation on heart rate variability: a controlled crossover trial. Journal of Manipulative and Physiological Therapeutics. 2006;29(8):603-610.

24. Driscoll MD, Hall MJ. Effects of spinal manipulative therapy on autonomic activity and the cardiovascular system: a case study using the electrocardiogram and arterial tonometry. J Manipulative Physiol Ther. 2000;23(8):545-50. 